1. KY Fan \& A. J. Hofrman, "Lower bounds for the rank and location of the eigenvalues of a matrix," Contributions to the Solution of Systems of Linear Equations and the Determination of Eigenvalues, edited by Olga Taussky, Nat. Bur. Standards. Appl. Math. Ser., v. 39, 1954, p. $117-130$.

2. David G. Feingold \& Richard S. VarGa, "Block diagonally dominant matrices and generalizations of the Gerschgorin Circle Theorem," Case Institute of Technology, Cleveland 6, Ohio, Report No. 1062, 1962.

3. A. S. Housenolder, "The approximate solution of matrix problems," J. Assoc. Comput. Mach., v. 5, 1958, p. 205-243.

\title{
Supplementary Data and Remarks Concerning a Hardy-Littlewood Conjecture
}

\section{By Daniel Shanks}

Let $P_{a}(N)$ be the number of primes of the form $n^{2}+a$ for $1 \leqq n \leqq N$, and let $\bar{\pi}_{a}(N)$ be the number of primes $\leqq N$ for which $-a$ is a quadratic nonresidue. In [1] we discussed a conjecture of Hardy and Littlewood to the effect that

$$
\frac{P_{a}(N)}{\bar{\pi}_{a}(N)} \sim h_{a}
$$

where the constant $h_{a}$ is given by

$$
h_{a}=\prod_{p \nmid a}\left(1-\left(\frac{-a}{p}\right) \frac{1}{p-1}\right),
$$

the product being taken over the odd primes $p$, with $(-a / p)$ the Legendre Symbol. We gave in [1] a heuristic argument in support of (1), a method of computing the $h_{a}$, and supporting empirical data for the six cases $a=1, \pm 2, \pm 3$, and 4 .

Subsequently the primes were also counted for six other cases, namely $a=$ $\pm 5, \pm 6, \pm 7$, and since such data are not available elsewhere it seems desirable to record them in a brief note. In Tables 1,2 , and 3 we show summaries for $N=$ $10,000(10,000) 180,000$ in the same format as the tables in [1].

While accurate values of $h_{a}$ in these six cases had not been computed, it was at once apparent that (1) is at least roughly correct for these values of $a$ also. Quite recently [2] tables of $L_{a}(s)$ for $a= \pm 6$ have been computed by J. W. Wrench, Jr., and, on the basis of these, one finds

$$
\begin{aligned}
h_{6} & =0.71304162 \\
h_{-6} & =1.03575587 .
\end{aligned}
$$

These are in good agreement with the empirical ratios in Table 2. Equally accurate constants for $a= \pm 5$ and \pm 7 are more difficult to compute, and are not yet available.

We may note the following:

1. Of the twelve forms, $n^{2}+a$, that we have investigated, $n^{2}+7$ has the most primes. Its (empirical) $h_{7}$, equal to 1.98 , indicates that numbers of this form are primes nearly twice as often as numbers of the same magnitude chosen at random.

Received November 8, 1962. 
2. On the other extreme, $n^{2}+\tilde{j}$ is a prime only about one-half as often $\left(h_{5}=0.53\right)$ as numbers of the same magnitude chosen at random.

3. And, of all twelve cases, $n^{2}-6$ is most nearly normal in number of primes, since $h_{-6}$ is the closest to 1 .

4. We also note that $n^{2}+6$ and $n^{2}+2$ have constants that are re-

TABLe 1

\begin{tabular}{c|c|c|c|c|c|c}
\hline$N$ & $P_{5}(N)$ & $\bar{\pi}_{5}(N)$ & $P_{5}(N) / \bar{\pi}_{5}(N)$ & $P_{-5}(N)$ & $\bar{\pi}_{-5}(N)$ & $P_{-5}(N) / \bar{\pi}_{-5}(N)$ \\
\hline 10,000 & 339 & 613 & 0.5530 & 1088 & 618 & 1.7605 \\
20,000 & 627 & 1136 & 0.5519 & 2051 & 1138 & 1.8023 \\
30,000 & 880 & 1622 & 0.5425 & 2916 & 1633 & 1.7857 \\
40,000 & 1123 & 2107 & 0.5330 & 3780 & 2112 & 1.7898 \\
50,000 & 1376 & 2589 & 0.5315 & 4593 & 2578 & 1.7816 \\
60,000 & 1606 & 3054 & 0.5259 & 5420 & 3038 & 1.7841 \\
70,000 & 1846 & 3500 & 0.5274 & 6214 & 3479 & 1.7861 \\
80,000 & 2099 & 3945 & 0.5321 & 7018 & 3932 & 1.7848 \\
90,000 & 2332 & 4389 & 0.5313 & 7834 & 4367 & 1.7939 \\
100,000 & 2567 & 4817 & 0.5329 & 8579 & 4813 & 1.7825 \\
110,000 & 2802 & 5238 & 0.5349 & 9344 & 5257 & 1.7774 \\
120,000 & 3028 & 5666 & 0.5344 & 10119 & 5671 & 1.7843 \\
130,000 & 3260 & 6090 & 0.5353 & 10858 & 6105 & 1.7785 \\
140,000 & 3493 & 6519 & 0.5358 & 11603 & 6524 & 1.7785 \\
150,000 & 3723 & 6954 & 0.5354 & 12341 & 6933 & 1.7800 \\
160,000 & 3936 & 7371 & 0.5340 & 13097 & 7350 & 1.7819 \\
170,000 & 4148 & 7763 & 0.5343 & 13844 & 7757 & 1.7847 \\
180,000 & 4368 & 8170 & 0.5346 & 14575 & 8182 & 1.7813 \\
\hline
\end{tabular}

TABLE 2

\begin{tabular}{c|c|c|c|c|c|c}
\hline \multicolumn{1}{c|}{$N$} & $P_{6}(N)$ & $\bar{\pi}_{6}(N)$ & $P_{6}(N) / \bar{\pi}_{6}(N)$ & $P_{-6}(N)$ & $\bar{\pi}_{-6}(N)$ & $P_{-6}(N) / \bar{\pi}_{-6}(N)$ \\
\cline { 1 - 3 } 10,000 & 444 & 616 & 0.7208 & 643 & 620 & 1.0371 \\
20,000 & 782 & 1147 & 0.6818 & 1155 & 1142 & 1.0114 \\
30,000 & 1147 & 1633 & 0.7024 & 1684 & 1635 & 1.0300 \\
40,000 & 1500 & 2125 & 0.7059 & 2164 & 2111 & 1.0251 \\
50,000 & 1834 & 2583 & 0.7100 & 2649 & 2565 & 1.0327 \\
60,000 & 2157 & 3049 & 0.7075 & 3134 & 3044 & 1.0296 \\
70,000 & 2488 & 3490 & 0.7129 & 3607 & 3476 & 1.0377 \\
80,000 & 2793 & 3919 & 0.7127 & 4086 & 3913 & 1.0442 \\
90,000 & 3123 & 4352 & 0.7176 & 4559 & 4363 & 1.0449 \\
100,000 & 3420 & 4795 & 0.7132 & 5010 & 4804 & 1.0429 \\
110,000 & 3733 & 5226 & 0.7143 & 5462 & 5238 & 1.0428 \\
120,000 & 4038 & 5650 & 0.7147 & 5913 & 5668 & 1.0432 \\
130,000 & 4352 & 6077 & 0.7161 & 6362 & 6097 & 1.0435 \\
140,000 & 4671 & 6516 & 0.7169 & 6801 & 6530 & 1.0415 \\
150,000 & 4978 & 6937 & 0.7176 & 7229 & 6953 & 1.0397 \\
160,000 & 5286 & 7346 & 0.7196 & 7656 & 7382 & 1.0371 \\
170,000 & 5580 & 7752 & 0.7198 & 8098 & 7793 & 1.0391 \\
180,000 & 5889 & 8160 & 0.7217 & 8552 & 8209 & 1.0418 \\
\hline
\end{tabular}


TABLE 3

\begin{tabular}{c|r|r|r|r|r|r}
\hline \multirow{2}{*}{$N$} & $P_{7}(N)$ & $\bar{\pi}_{7}(N)$ & $P_{7}(N) / \bar{\pi}_{7}(N)$ & $P_{-7}(N)$ & $\bar{\pi}_{-7}(N)$ & $P_{-7}(N) / \bar{\pi}_{-7}(N)$ \\
& & & & & \\
10,000 & 1238 & 620 & 1.9968 & 440 & 616 & 0.7143 \\
20,000 & 2254 & 1134 & 1.9877 & 841 & 1145 & 0.7345 \\
30,000 & 3225 & 1638 & 1.9689 & 1226 & 1628 & 0.7531 \\
40,000 & 4176 & 2110 & 1.9792 & 1590 & 2112 & 0.7528 \\
50,000 & 5094 & 2569 & 1.9829 & 1937 & 2563 & 0.7558 \\
60,000 & 6004 & 3035 & 1.9783 & 2272 & 3026 & 0.7508 \\
70,000 & 6891 & 3481 & 1.9796 & 2617 & 3479 & 0.7522 \\
80,000 & 7788 & 3948 & 1.9726 & 2958 & 3944 & 0.7500 \\
90,000 & 8697 & 4372 & 1.9893 & 3304 & 4389 & 0.7528 \\
100,000 & 9521 & 4813 & 1.9782 & 3627 & 4828 & 0.7512 \\
110,000 & 10419 & 5250 & 1.9846 & 3977 & 5252 & 0.7572 \\
120,000 & 11228 & 5669 & 1.9806 & 4291 & 5675 & 0.7561 \\
130,000 & 12070 & 6101 & 1.9784 & 4632 & 6109 & 0.7582 \\
140,000 & 12904 & 6523 & 1.9782 & 4953 & 6521 & 0.7596 \\
150,000 & 13739 & 6943 & 1.9788 & 5258 & 6943 & 0.7573 \\
160,000 & 14580 & 7360 & 1.9810 & 5585 & 7362 & 0.7586 \\
170,000 & 15450 & 7765 & 1.9897 & 5914 & 7770 & 0.7611 \\
180,000 & 16240 & 8198 & 1.9810 & 6225 & 8211 & 0.7581 \\
\hline
\end{tabular}

markably close:

$$
\begin{aligned}
& h_{6}=0.71304162 \\
& h_{2}=0.71306310 .
\end{aligned}
$$

Corresponding to 100,000 primes of the former type, there should be 100,003 primes of the latter. Up to $N=180,000$ the two classes take turns being in the lead, but, if (1) is true, $n^{2}+2$ must eventually take, and hold, the lead. A reasonable estimate, however, suggests that this could be postponed until $N$ exceeds $3 \cdot 10^{10}$.

5. We show in Figure 1 a bar graph of $h_{a}$ for $a=-20$ (1) 9 . These include the previously computed values; the present computed values in equation (3) above and Table 4 below; the empirical values in comments 1 and 2 above; and the easily computed $h_{-9}=h_{-16}=0, h_{8}=h_{2}, h_{-8}=h_{-2}, h_{-12}=h_{-3}, h_{-20}=h_{-5}, h_{9}=\frac{2}{3} h_{1}$, and $h_{-18}=\frac{2}{3} h_{-2}$. One sees at a glance that the distribution of primality in the neighborhood of square numbers is anything but uniform.

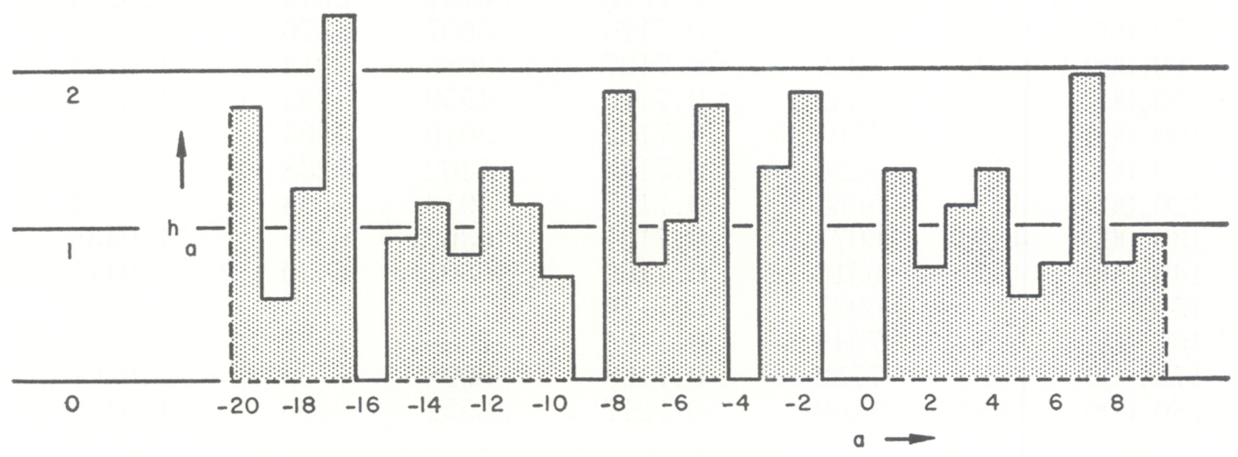

FIg. 1-Distribution of primality in the neighborhood of square numbers. 
6. Next, two remarks concerning the computation of $h_{a}$. If $a<0$, both $L_{a}$ (1) and $L_{a}(2)$ are available in closed form [2]. Thus, one may calculate $h_{a}$ with moderate accuracy quite simply by the use of $[1$, eq. (18)]. The covergence of the remaining factor is fairly rapid, and particularly so if $-a$ is a quadratic residue of only a few small primes.

For example, up to $p=43,(17 / p)=+1$ only for 13 and 19 . Thus, using the theory in [1] and [2], we determine that

$$
h_{-17}=\frac{145 \pi^{2}}{289 \log (4+\sqrt{17})}\left(1-\frac{2}{13 \cdot 12^{2}}\right)\left(1-\frac{2}{19 \cdot 18^{2}}\right) \prod_{\substack{p \geq 43 \\\left(\frac{17}{p}\right)=+1}}\left(1-\frac{2}{p \cdot(p-1)^{2}}\right) .
$$

Ignoring the last factor we obtain $h_{-17} \approx 2.3606$ and therefore

$$
P_{-17}(N) \sim 1.1803 \int_{2}^{N} \frac{d n}{\log n} .
$$

(The Hardy-Littlewood conjecture is particularly frustrating in a case such as this, where the sequence $n^{2}-17$ has even more primes than the sequence $n$, since we are unable to prove that $P_{-17}(N) \rightarrow \infty$ even in the weakest possible way.)

By similar computations we can compute three decimal place values of $h_{a}$ for other negative $a$, and we present some such values in Table 4 .

TABLE 4

\begin{tabular}{|l|l|}
\hline$h_{-5}=1.773(3)$ & $h_{-14}=1.151(7)$ \\
$h_{-7}=0.757(4)$ & $h_{-15}=0.911(8)$ \\
$h_{-10}=0.671(1)$ & $h_{-17}=2.360(6)$ \\
$h_{-11}=1.148(0)$ & $h_{-19}=0.544(2)$ \\
$h_{-13}=0.807(2)$ & \\
\hline
\end{tabular}

7. There also is an interesting complementary formula for $h_{a}$. Here the convergence is fastest if $-a$ is a quadratic residue for many small primes. Since, by (2) we have

$$
h_{a}=\prod_{p \nmid a}\left(1-\left(\frac{-a}{p}\right) \frac{1}{p-1}\right)
$$

we now define

$$
h_{a}^{*}=\prod_{p \nmid a}\left(1+\left(\frac{-a}{p}\right) \frac{1}{p-1}\right) .
$$

Then

$$
h_{a} h_{a}^{*} \prod_{p \nmid a}\left(1-\frac{1}{(p-1)^{2}}\right)=\prod_{p>2}\left(1-\frac{1}{(p-1)^{2}}\right)=c_{2} .
$$

Since $c_{2}$, the so-called "twin-prime constant," is accurately known [3], we may compute $h_{a}{ }^{*}$ by (4), and utilize (5) to evaluate $h_{a}$. In this way we interchange the roles of the quadratic residues and nonresidues. Using the notation of [1] we 
therefore obtain

$$
h_{a}=\frac{0.6601618158}{\prod_{p_{\perp} a}\left(1-\frac{1}{(p-1)^{2}}\right) L_{a}(1)} \prod_{s=2}^{\infty}\left(\frac{\zeta_{a}(s)}{L_{a}(s)}\right)^{b(s)}
$$

We note that the right side of (6) converges monotonically increasing, while that in $\left[1\right.$, eq. (18)] converges monotonically decreasing. Thus $h_{a}$ may be bounded.

To illustrate $(6)$, and such bounds, we note that $(19 / q)=-1$ for $q=7,11$, 13,23 , while $(19 / p)=+1$ for $p=3,5$, and 17 . Therefore we find

$$
\begin{aligned}
h_{-19}=\frac{0.6601618158}{\log (170+39 \sqrt{19})} \cdot \frac{29160}{6137} \cdot \frac{252}{250} \cdot \frac{1100}{1098} \cdot \frac{1872}{1870} \cdot \frac{11132}{11130} \\
\cdot \prod_{\substack{q \geq 29 \\
\left(\frac{19}{q}\right)=-1}}\left(1-\frac{2}{q(q-1)^{2}}\right)^{-1} .
\end{aligned}
$$

Ignoring the last factor, we obtain

$$
0.54411<h_{-19} .
$$

On the other hand, from [1, eq. (18)], we have

$h_{-19}=\frac{\pi^{2}}{\log (170+39 \sqrt{19})} \cdot \frac{2715}{6859} \cdot \frac{10}{12} \cdot \frac{78}{80} \cdot \frac{4350}{4352} \cdot \prod_{\substack{p \geq \geqq 31 \\\left(\frac{19}{p}\right)=+1}}\left(1-\frac{2}{p(p-1)^{2}}\right)$,

and therefore

$$
h_{-19}<0.54431 \text {. }
$$

8. Finally, these bounds suggest a weakened version of the Hardy-Littlewood conjecture that may be less unattainable. We have, for all $a$,

$$
\frac{c_{2}}{L_{a}(1)}<h_{a}<\frac{\pi^{2}}{8 L_{a}(1)}
$$

or, with numerical coefficients,

$$
\frac{0.6601618158}{L_{a}(1)}<h_{a}<\frac{1.233700550}{L_{a}(1)} .
$$

While these are not very close bounds, they are valid for all $a$, and they suggest, for sufficiently large $N$, the inequalities:

$$
\frac{c_{2}}{2 L_{a}(1)} \int_{2}^{N} \frac{d n}{\log n}<P_{a}(N)<\frac{\pi^{2}}{16 L_{a}(1)} \int_{2}^{N} \frac{d n}{\log n} .
$$

The best coefficients that have been proven are $2 h_{a}$ on the right and 0 on the left. For the former, see the use, by Bateman and Stemmler [4], of A. Selberg's sieve method. Equation (8) is, of course, reminiscent of the old Chebyshev inequalities for $\pi(N)$, and historical precedent therefore suggests that an investigation of such 
bounds is in order. While the lower bound would be particularly important, the improved upper bound would also be useful.

Applied Mathematics Laboratory

David Taylor Model Basin

Washington 7, D. C.

1. Daniel Shanks, "On the conjecture of Hardy \& Littlewood concerning the number of primes of the form $n^{2}+a$, , Math. Comp., v. 14, 1960, p. 321-332.

2. Daniel Shanks \& John W. Wrench, JR., "The calculation of certain Dirichlet series," Math. Comp., v. 17, 1963, p. 136-154.

3. JoHn W. Wrench, JR., "Evaluation of Artin's constant and the twin-prime constant," Math. Comp., v. 15, 1961, p. 396-398.

4. Paul T. Bateman \& Rosemarie M. Stemmler, "Waring's problem in algebraic number fields and primes of the form $\left(p^{r}-1\right) /\left(p^{d}-1\right)$," Illinois J. Math., v. 6, 1962, p. 142-156.

\section{An Approximation to the Fermi Integral $F_{1 / 2}(x)$}

\section{By H. Werner and G. Raymann}

The Fermi Integral as defined, for instance, in the Handbuch der Physik, Bd. $\mathrm{XX}$, S. 58 [1], is given by

$$
F_{p}(x)=\int_{0}^{\infty} \frac{t^{p}}{e^{t-x}+1} d t
$$

The function $F_{1 / 2}(x)$ has for negative values of $x$ an expansion of the form

$$
F_{1 / 2}(x)=\frac{\sqrt{\pi}}{2} \sum_{\nu=1}^{\infty}(-1)^{\nu-1} \cdot \frac{e^{\nu x}}{\nu^{3 / 2}},
$$

and for large positive $x$ the asymptotic expansion

$$
\begin{aligned}
F_{1 / 2}(x) \sim x^{3 / 2}\left[\frac{2}{3}+\frac{\pi^{2}}{12 \cdot x^{2}}+\left(\begin{array}{c}
\frac{1}{2} \\
3
\end{array}\right)\right. & \cdot \frac{7}{60} \cdot \frac{\pi^{4}}{x^{4}}+\cdots \\
& \left.+\left(\begin{array}{c}
\frac{1}{2}-1 \\
2 n-1
\end{array}\right) \frac{2^{2 n-1}-1}{n}\left|B_{2 n}\right| \cdot \frac{\pi^{2 n}}{x^{2 n}}+\cdots\right]
\end{aligned}
$$

compare [2], formulas (10) and (12);

$B_{2 n}$ are the Bernoulli numbers, given for example in [3], page 298. We obtained Chebyshev approximations to $F_{1 / 2}(x)$, based upon the table by McDougall and Stoner [4]. This table was subtabulated by interpolation with a fifth-degree polynomial. The approximations are

$$
\begin{array}{ll}
F_{1 / 2}^{*}(x)=e^{x} \sum_{\nu=0}^{5} a_{\nu} e^{\nu x} & \text { for }-\infty<x \leqq+1, \\
F_{1 / 2}^{*}(x)=x^{3 / 2}\left[\frac{2}{3}+\sum_{\nu=0}^{5} \frac{b_{\nu}}{x^{2 \nu+2}}\right] & \text { for }+1<x<+\infty,
\end{array}
$$

Received March 27, 1961. This note was prepared at the AEG Forschungs-Institut, Frankfurt (Main). 